\title{
A novel two-stage estimation algorithm for nonlinear Hammerstein-Wiener systems from noisy input and output data
}

\author{
Ziyun Wang*, Zhicheng Ji \\ Key Laboratory of Advanced Process Control for Light Industry (Ministry of Education), Jiangnan University, Wuxi 214122, \\ PR China
}

\begin{abstract}
This paper investigates the identification problem of Hammerstein-Wiener errors-in-variable systems where the measurement errors of the system input and output are either temporally white or have relatively short memory size compared to the data length, but the corresponding variances are unknown. A two-stage algorithm is developed to estimate the unknown parameters with the first stage employing a modified bias-eliminating least squares algorithm, followed by a singular value decomposition in the second stage. Our proposed estimator is shown to be asymptotically unbiased. The simulation result shows the effectiveness of the proposed algorithm.
\end{abstract}

Key words: Bias-eliminating least squares, Singular value decomposition, Hammerstein-Wiener system, System identification

\section{Introduction}

System identification is an important branch of system control field that attracts researchers greatly concern $[1,2,3,4,5]$. For example, Wang and Ding derived an auxiliary model-based multi-innovation generalized extended stochastic gradient algorithm for modelling the Box-Jenkins systems by using the multi-innovation identification theory, and analyzed the convergence of the estimation algorithm based on the stochastic martingale theory [6]; Xiong et al. studied the problem of parameter estimation for nonlinear Wiener systems in the stochastic framework and proposed an expectation-maximization algorithm considering the randomly missing outputs [7]; Zhang et al. presented an auxiliary model based method to replace the unmeasurable outputs in the information vector of the Errors-in-Variables system by auxiliary model outputs [8].

The least squares (LS for short) method is one of the most widely used approaches to solve the system identification problem. The well known books of Söderström ${ }^{[9]}$ and Ljung ${ }^{[10]}$ provide treatises for estimating parameters of different models. However the estimates derived from the LS algorithm are biased in general when both input and output involve measurement errors, even if the errors are white and uncorrelated. For this reason EIV (Errors-in-Variables) models have received great attention from the system identification community. See for example, the work of Huffel ${ }^{[11]}$, Huffel and Lemmerling ${ }^{[12]}$, Mu and Chen ${ }^{[13]}$, Söderström, Irshad, Mossberg and Zheng ${ }^{[14]}$, and Song ${ }^{[15]}$. In [16], Söderström provides the background and motivations of the EIV model, then Söderström, Mossberg and Hong ${ }^{[17]}$ propose a covariance matching approach for identifying dynamic EIV models. These active researches on EIV models lead to extensive study of the bias-eliminating least squares (BELS) algorithm that is asymptotically unbiased, assuming independent and identical distribution (i.i.d.) for the input and output measurement errors.

The developments in EIV model identification are notwithstanding. There lacks similar activities in developing the BELS algorithm for identifying nonlinear models due to probably the nonlinear input/output relation of the measurement data that obscures the nature of the underlying estimation problem under unknown noise covariance. In the LS front, Bai ${ }^{[18]}$ studies the corresponding identification problem for the Hammerstein-Wiener system, and presents an identification method. His method estimates the system parameters in two steps: one is the recursive LS algorithm and the other adopts the SVD idea. However, the proposed method in [18] is biased for the nonlinear EIV system.

The main contribution of this paper is listed as follows:

\footnotetext{
This work was supported in part by the Fundamental Research Funds for the Central Universities (No. JUSRP115A30).

* Corresponding author

Email addresses: wangzy0601@163.com (Ziyun Wang), zcji123@126.com (Zhicheng Ji)
} 
- An interesting result obtained in this paper is that without any assumption on the noise variances, the BELS solution can be modified to provide an asymptotically unbiased estimate for identifying nonlinear EIV systems, provided that the McMillian degree of the linear part is known and the nonlinear terms are specified, compared with the published algorithms in [18].

- It is proved that the proposed modified BELS solution converges strongly to the true system parameters, i.e., with probability 1 (w.p.1) as the number of measurements approaches infinity under the condition of persist excitation and the presented algorithm is easy to use.

- The provided simulation also shows that with the same $\sigma^{2}$, the proposed modified BELS algorithm can obtain more accurate parameter estimates than the LS identification algorithm.

The paper is organized as follows. Section 2 provides preliminaries on the standard EIV estimation problem and the reformulation of the EIV system identification for the Hammerstein-Wiener systems. Section 3 is focused on deriving the BELS algorithm with modification in two stages and presents the proof for the asymptotically unbiased property of our proposed BELS algorithm in identifying the Hammerstein-Wiener EIV system where the strong consistency is shown to hold. Section 4 illustrates the effectiveness of the proposed algorithm via simulation example. The paper is concluded in Section 5 .

\section{Problem formulation}

Let $\mathbb{R}$ be the set of real numbers. Denote $\mathbb{R}^{n}$ and $\mathbb{R}^{n \times m}$ as the sets of real vectors of dimension $n$ and real matrices of dimension $n \times m$, respectively. For matrix $X$, its transpose is denoted by $X^{\mathrm{T}}$.

Let $\Theta \in \mathbb{R}^{p \times n}$ be the parameter matrix of interest satisfying

$$
\Theta X=Y, X \in \mathbb{R}^{n \times N}, Y \in \mathbb{R}^{p \times N}
$$

where $N>n+p$. In practice the precise values of the input-output data $X$ and $Y$ are unknown. Instead only their measurements, denoted by $\hat{X}$ and $\hat{Y}$, respectively, are available, giving rise to the EIV model

$$
\left[\begin{array}{l}
\hat{Y} \\
\hat{X}
\end{array}\right]=\left[\begin{array}{l}
Y \\
X
\end{array}\right]+\Delta
$$

where elements of $\Delta$ are i.i.d. (identically and independently distributed) random variables. The true system of equation in (1) is called EIV model. The nonlinear system identification problem to be studied in this paper is described in Figure 1 below.

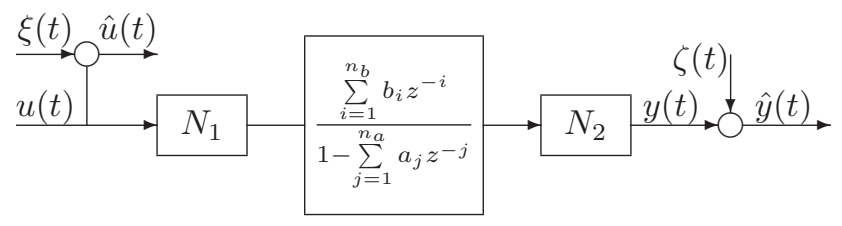

Figure 1: Hammerstein-Wiener EIV System model with noisy measurement data

The nonlinear system in Fig. 1 is termed as Hammerstein-Wiener EIV model governed by the following nonlinear difference equation

$$
y(t)=\sum_{i=1}^{q} a_{i}\left\{\sum_{l=1}^{m} d_{l} g_{l}[y(t-i)]\right\}+\sum_{j=1}^{p} b_{j}\left\{\sum_{k=1}^{n} c_{k} f_{k}[u(t-j)]\right\}
$$

where $u(t)$ and $y(t)$ are the true input and output at time index $t$, respectively. The observed input and output signals $\hat{u}(t)$ and $\hat{y}(t)$ are described by the following EIV relation:

$$
\left[\begin{array}{l}
\hat{y}(t) \\
\hat{u}(t)
\end{array}\right]=\left[\begin{array}{l}
y(t) \\
u(t)
\end{array}\right]+\varepsilon(t), \varepsilon(t)=\left[\begin{array}{l}
\zeta(t) \\
\xi(t)
\end{array}\right]
$$

where $\zeta(t)$ is the measurement noise at the output and $\xi(t)$ the measurement noise at the input at time index $t$. There is no assumption on i.i.d. for the measurement errors $\zeta(t)$ and $\xi(t)$, nor the knowledge of the variances. 
However we do assume that the measurement errors are temporally white. If temporally whiteness does not hold, we assume that the memory length of the measurement errors is small relative to the data length $N$.

Define $\boldsymbol{a}=\left[\begin{array}{lll}a_{1} & \cdots & a_{q}\end{array}\right]^{\mathrm{T}}, \boldsymbol{b}=\left[\begin{array}{lll}b_{1} & \cdots & b_{p}\end{array}\right]^{\mathrm{T}}, \boldsymbol{c}=\left[\begin{array}{lll}c_{1} & \cdots & c_{n}\end{array}\right]^{\mathrm{T}}$ and $\boldsymbol{d}=\left[\begin{array}{lll}d_{1} & \cdots & d_{m}\end{array}\right]^{\mathrm{T}}$. The purpose of this paper is to derive an asymptotically unbiased algorithm, similar to the BELS for linear systems, for identify parameter vectors $\boldsymbol{a}, \boldsymbol{b}, \boldsymbol{c}$ and $\boldsymbol{d}$ of the Hammerstein-Wiener model in (3).

\section{The BELS based two-stage algorithm}

Identification of EIV systems has been studied via the bias eliminating LS algorithm in the past. Our goal is to develop a similar algorithm that fits more naturally the Hammerstein-Wiener system identification in the absence of the knowledge of the noise covariances. Note that any pair of $\left(a_{i}, d_{l}\right)$ and $\left(b_{j}, c_{k}\right)$ are not unique in model (3). It is essential to have an uniqueness assumption.

Assumption $\mathbf{1}^{[18]}$ Assume that $\|\boldsymbol{a}\|=1$ and $\|\boldsymbol{b}\|=1$ with $\|\cdot\|$ the Euclidean norm and the sign of the first nonzero element of $\boldsymbol{a}$ and $\boldsymbol{b}$ being positive.

Under Assumption 1, it can be easily verified that the parameterization of system (3) is unique. Define

$$
\begin{aligned}
& \boldsymbol{\theta}:=\left[\begin{array}{llllllllll}
b_{1} c_{1} & \cdots & b_{1} c_{n} & \cdots & b_{p} c_{n} & a_{1} d_{1} & \cdots & a_{1} d_{m} & \cdots & a_{q} d_{m}
\end{array}\right] \\
& =\left[\begin{array}{lllll}
\theta_{1} & \cdots & \theta_{p n} & \cdots & \theta_{p n+q m}
\end{array}\right] \in \mathbb{R}^{1 \times(p n+q m)}, \\
& \Theta_{b c}:=\boldsymbol{b c}^{\mathrm{T}}=\left[\begin{array}{cccc}
b_{1} c_{1} & b_{1} c_{2} & \cdots & b_{1} c_{n} \\
b_{2} c_{1} & b_{2} c_{2} & \cdots & b_{2} c_{m} \\
\vdots & \vdots & \ddots & \vdots \\
b_{p} c_{1} & b_{p} c_{2} & \cdots & b_{p} c_{n}
\end{array}\right] \in \mathbb{R}^{p \times n}, \\
& \boldsymbol{\Theta}_{a d}:=\boldsymbol{a d}^{\mathrm{T}}=\left[\begin{array}{cccc}
a_{1} d_{1} & a_{1} d_{2} & \cdots & a_{1} d_{m} \\
a_{2} d_{1} & a_{2} d_{2} & \cdots & a_{2} d_{m} \\
\vdots & \vdots & \ddots & \vdots \\
a_{q} d_{1} & a_{q} d_{2} & \cdots & a_{q} d_{m}
\end{array}\right] \in \mathbb{R}^{q \times m} .
\end{aligned}
$$

Let the generalized regressor be defined as

$$
\begin{aligned}
\phi(t):=[ & f_{1}(u(t-1)) \cdots f_{n}(u(t-1)) \cdots f_{1}(u(t-p)) \\
& \cdots f_{n}(u(t-p)) g_{1}(y(t-1)) \cdots g_{m}(y(t-1)) \\
& \left.\cdots g_{1}(y(t-q)) \cdots g_{m}(y(t-q))\right]^{\mathrm{T}} \in \mathbb{R}^{p n+q m} .
\end{aligned}
$$

Model (3) can be rewritten as $y(t)=\boldsymbol{\theta} \boldsymbol{\phi}(t)$, i.e.,

$$
\left[\begin{array}{ll}
-1 \boldsymbol{\theta}
\end{array}\right] \boldsymbol{\psi}(t)=0, \quad \boldsymbol{\psi}(t)=\left[\begin{array}{l}
y(t) \\
\phi(t)
\end{array}\right] .
$$

For the given $\{\hat{u}(t), \hat{y}(t)\}_{t=1}^{N}$, the measurement errors in $\boldsymbol{\psi}(t)$ and $\boldsymbol{\psi}(t-M)$ are uncorrelated, provided that $M \geq p n+q m$, assuming temporarily that the measurement errors are white. We set $M=p n+q m$ due to the whiteness assumption in this section. Denote

$$
\hat{R}_{\hat{\psi}}(M):=\frac{1}{M} \sum_{t=M+1}^{N} \hat{\boldsymbol{\psi}}(t) \hat{\boldsymbol{\psi}}(t-M)^{\prime}
$$

as the sampled second order statistics where $\hat{\boldsymbol{\psi}}(t)$ is the measurements of $\boldsymbol{\psi}(t)$ at time $t$. Assume

$$
\operatorname{rank}\left\{\frac{1}{M} \sum_{t=M+1}^{N} \boldsymbol{\psi}(t) \boldsymbol{\psi}(t-M)^{\prime}\right\}=M
$$

in absence of noises. Define the performance index

$$
J(\hat{\boldsymbol{\theta}} ; M):=\frac{1}{1+\|\hat{\boldsymbol{\theta}}\|^{2}}\left\|[-1 \quad \hat{\boldsymbol{\theta}}] \hat{R}_{\hat{\psi}}(M)\right\|^{2} .
$$

Our proposed algorithm departs the conventional BELS in that it minimizes $J(\hat{\boldsymbol{\theta}} ; M)$ in obtaining the estimate $\hat{\boldsymbol{\theta}}$. Recall that the known existing BELS algorithm minimizes $J(\hat{\boldsymbol{\theta}} ; 0)$ that requires the known error variances 
or unknown but equal error variances of the input and output measurement data. Let $\hat{R}_{\hat{\psi}}(M)=U S V^{\prime}$ be the SVD where $U=\left[\boldsymbol{u}_{1}, \boldsymbol{u}_{2}, \cdots, \boldsymbol{u}_{M+1}\right]$ with $\boldsymbol{u}_{M+1}$ the left singular vector corresponding to the smallest singular value. The minimizer of $J(\hat{\boldsymbol{\theta}} ; M)$ is given by

$$
\hat{\boldsymbol{\theta}}(N)=-\frac{1}{\boldsymbol{u}_{1, M+1}}\left[\begin{array}{c}
\boldsymbol{u}_{2, M+1} \\
\boldsymbol{u}_{3, M+1} \\
\vdots \\
\boldsymbol{u}_{M+1, M+1}
\end{array}\right]
$$

where $M=p n+q m$ and

$$
\boldsymbol{u}_{M+1}:=\left[\begin{array}{c}
\boldsymbol{u}_{1, M+1} \\
\boldsymbol{u}_{2, M+1} \\
\vdots \\
\boldsymbol{u}_{M+1, M+1}
\end{array}\right]
$$

This procedure outlines the first step of our modified two-stage algorithm from the one in [18], and provides the modified BELS estimate $\hat{\boldsymbol{\theta}}(N)$. Denote $\hat{\boldsymbol{a}}(N), \hat{\boldsymbol{b}}(N), \hat{\boldsymbol{c}}(N), \hat{\boldsymbol{d}}(N), \hat{\boldsymbol{\Theta}}_{b c}(N)$ and $\hat{\boldsymbol{\Theta}}_{a d}(N)$ as the estimate of $\boldsymbol{a}, \boldsymbol{b}, \boldsymbol{c}, \boldsymbol{d}, \boldsymbol{\Theta}_{b c}$ and $\boldsymbol{\Theta}_{a d}$, respectively, which are related to entries of $\hat{\boldsymbol{\theta}}(N)$. The following describes the second step of the two-stage algorithm:

- Construct $\hat{\boldsymbol{\Theta}}_{a d}(N)$ and $\hat{\boldsymbol{\Theta}}_{b c}(N)$ from $\hat{\boldsymbol{\theta}}(N)$, and let

$$
\begin{aligned}
& \hat{\boldsymbol{\Theta}}_{b c}(N)=\sum_{i=1}^{\min (p, n)} \rho_{i} \boldsymbol{\tau}_{i} \boldsymbol{\iota}_{i}^{\mathrm{T}}, \\
& \hat{\boldsymbol{\Theta}}_{a d}(N)=\sum_{j=1}^{\min (q, m)} \sigma_{j} \boldsymbol{\mu}_{j} \boldsymbol{\nu}_{j}^{\mathrm{T}}
\end{aligned}
$$

be SVD of $\hat{\boldsymbol{\Theta}}_{b c}(N)$ and $\hat{\boldsymbol{\Theta}}_{a d}(N)$, where $\left\{\boldsymbol{\tau}_{i}\right\}_{i=1}^{p},\left\{\boldsymbol{\iota}_{i}\right\}_{i=1}^{n},\left\{\boldsymbol{\mu}_{i}\right\}_{i=1}^{q}$, and $\left\{\boldsymbol{\nu}_{i}\right\}_{i=1}^{m}$ are $p-, n-, q^{-}$, and $m$ dimensional orthogonal vectors, respectively.

- Let $s_{\tau}$ and $s_{\mu}$ denote the sign of the first nonzero element of $\tau_{1}$ and $\mu_{1}$, define the estimates

$$
\begin{aligned}
& \hat{\boldsymbol{b}}(N)=s_{\tau} \boldsymbol{\tau}_{1}, \hat{\boldsymbol{c}}(N)=s_{\tau} \rho_{1} \iota_{1}, \\
& \hat{\boldsymbol{a}}(N)=s_{\mu} \boldsymbol{\mu}_{1}, \hat{\boldsymbol{d}}(N)=s_{\mu} \sigma_{1} \boldsymbol{\nu}_{1} .
\end{aligned}
$$

The above second stage is identical to that of [18]. The next result shows the asymptotic unbiased property of the modified BELS algorithm when the elements of $\varepsilon(t)$ in (4) are unknown.

Theorem1 Consider system (3). Assume the rank condition in (7), and that measurement noises are white but with unknown noise variances. Then, the estimate $\hat{\boldsymbol{\theta}}$ computed by the two-stage identification algorithm is asymptotically unbiased for the case of $M=p n+q m$, i.e., $\hat{\boldsymbol{\theta}} \rightarrow \boldsymbol{\theta}$ as $N \rightarrow \infty$.

Proof: Define $\hat{\psi}(t)$ as the noise measurements for $1 \leqslant t \leqslant N$. Let $\hat{R}_{\hat{\psi}}$ be the sampled second order statistics as in (6). By $M=p n+q m$, the measurement errors in $\psi(t)$ and $\psi(t-M)$ are uncorrelated in light of the whiteness assumption. It follows that as $N \rightarrow \infty$, the noise term disappears asymptotically, and

$$
\hat{R}_{\hat{\psi}}(M) \rightarrow R_{\psi}(M)=\mathrm{E}\{\psi(t) \psi(t-M)\}
$$

as $N \rightarrow \infty$ where $M=p n+q m$.

Remark 1 The whiteness assumption can be removed, if the measurement errors have finite memory $\mu$, provided that $N-\mu>>1$. Indeed in this case, $M=\mu+p n+q m$ can be used, and thus $\boldsymbol{\psi}(t)$ and $\boldsymbol{\psi}(t-M)$ are again uncorrelated. It is important to indicate that if $M=\mu+p n+q m$ is close to $N$, then $\hat{R}_{\hat{\psi}}(M)$ can be very inaccurate, negatively impacting the identification performance. 


\section{An illustrative simulation example}

To illustrate the effectiveness of our modified BELS identification algorithm for the Hammerstein-Wiener system, we consider the same nonlinear system model from [18]:

$$
\begin{aligned}
& y(t)=a_{1}\left[d_{1} y(t-1)+d_{2} \sin (y(t-1))\right] \\
& +b_{1}\left[c_{1} u(t-1)+c_{2} u^{2}(t-1)\right] \\
& +b_{2}\left[c_{1} u(t-2)+c_{2} u^{2}(t-2)\right], \\
& \boldsymbol{\theta}=\left[a_{1}, b_{1}, b_{2}, c_{1}, c_{2}, d_{1}, d_{2}\right]^{\mathrm{T}} \\
& =[1,0.4472,0.8944,2.8,0.2,-0.5,0.2]^{\mathrm{T}} .
\end{aligned}
$$

In our simulation study, the input and output measurements $\{\hat{u}(t), \hat{y}(t)\}$ are the same as in (4) with

$$
\begin{aligned}
u(t)= & 2 \sin (2 t)+2 \sin (4 t)+0.15 \sin (6 t) \\
& +0.15 \sin (8 t)+0.1 \sin (10 t),
\end{aligned}
$$

and $\xi(t)$ and $\zeta(t)$ are white noise sequences with zero mean and variance $\sigma^{2}=0.1^{2}$. Applying our modified BELS algorithm and LS algorithm from [18] to estimate the parameters of this Hammerstein-Wiener EIV system, the relative estimation errors

$$
\delta:=\sqrt{\frac{1}{L} \sum_{i=1}^{L} \frac{\left\|\hat{\boldsymbol{\theta}}_{(i)}-\boldsymbol{\theta}\right\|^{2}}{\|\boldsymbol{\theta}\|^{2}}}
$$

versus $t$ are computed and plotted in Figure 2 below:

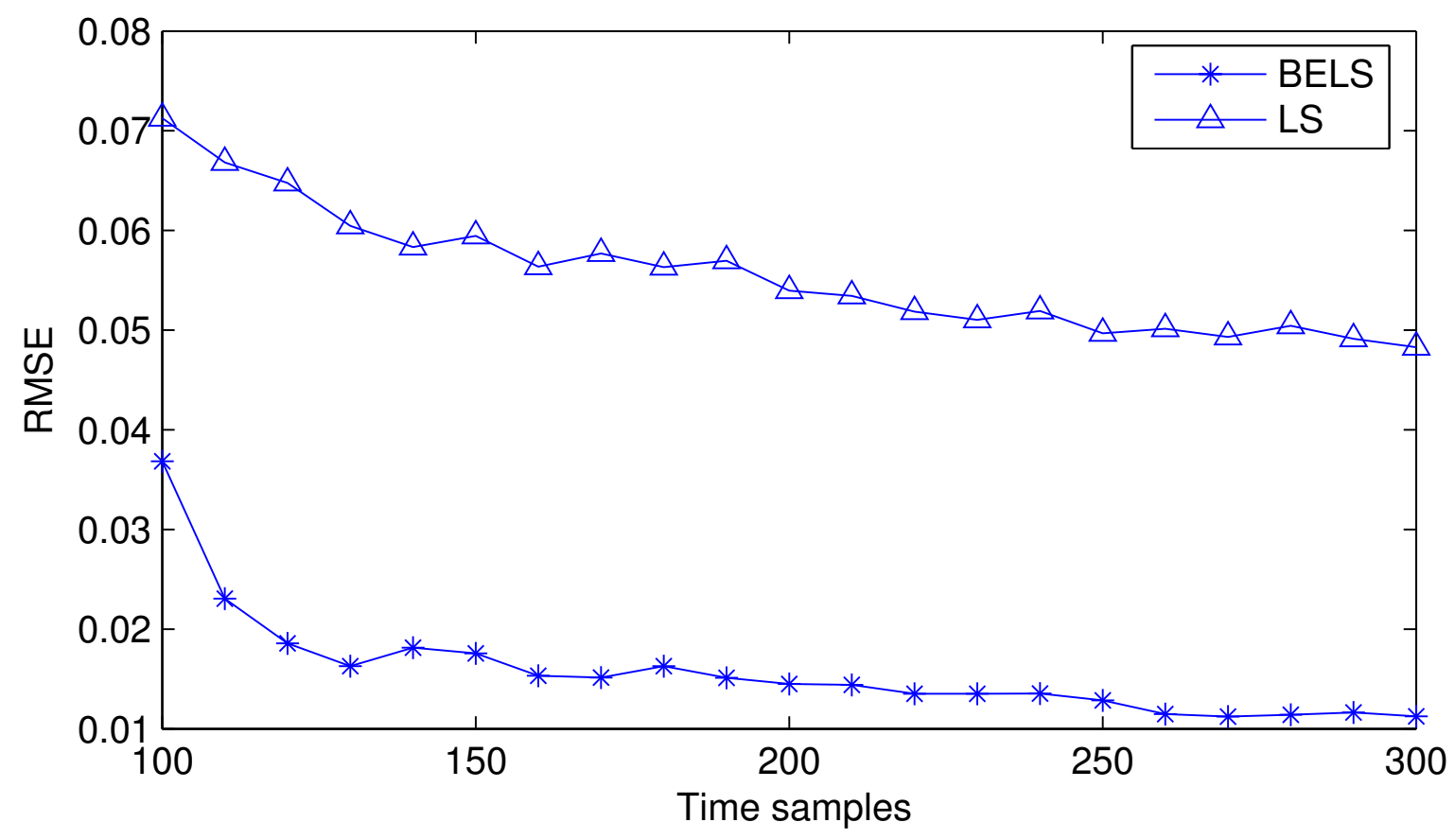

Figure 2: Relative RMSE using 1000 ensembles

The simulation results show that as $N$ increases, the RMSE value decreases. Compared with the LS algorithm proposed in [18], our modified BELS algorithm has a better performance, owing to the bias elimination nature.

\section{Conclusions}

This paper investigates the identification problem of nonlinear systems in the Hammerstein-Wiener form, and proposes a modified two-stage algorithm based on the modified BELS algorithm adapted from the linear case. Our work provides the proof for the property of the asymptotically unbiased estimate of our proposed 
algorithm, and is relatively easy to use, even if the noise variances at the input and output are unknown. While the whiteness of the measurement errors are assumed, it can be removed, provided that the measurement errors have finite memory with memory length relatively small compared with the data length. This method can also be used in solving the parameter estimation problem of other nonlinear systems [19, 20], such as Hammerstein and Wiener systems [21, 22, 23], block-oriented nonlinear systems [24, 25, 26].

\section{References}

[1] F. Ding. System Identification-New Theory and Methods, Science Press, Beijing, 2013

[2] F. Ding. System Identification-Performances Analysis for Identification Methods, Science Press, Beijing, 2014

[3] Y. Shi, T.W. Chen. Optimal design of multichannel transmultiplexers with stopband energy and passband magnitude constraints. IEEE Transactions on Circuits and Systems II-Analog and Digital Signal Processing, 2003, 50(9): 659-662.

[4] D. Liu, Y. Yang, Y. Zhang. Robust fault estimation for singularly perturbed systems with Lipschitz nonlinearity. Journal of the Franklin Institute, 2016, 353(4): 876-890.

[5] T. Du, L. Guo. Unbiased information filtering for systems with missing measurement based on disturbance estimation. Journal of the Franklin Institute, 2016, 353(4): 936-954.

[6] X.H. Wang, F. Ding. Convergence of the auxiliary model-based multi-innovation generalized extended stochastic gradient algorithm for Box-Jenkins systems. Nonlinear Dynamics, 2015, 82(1-2): 269-280.

[7] W.L. Xiong, X.Q. Yang, L. Ke, B.G. Xu. EM algorithm-based identification of a class of nonlinear Wiener systems with missing output data. Nonlinear Dynamics, 2015, 80(1-2): 329-339.

[8] Y. Zhang, Z. Zhao, G.M. Cui. Auxiliary model method for transfer function estimation from noisy input and output data. Applied Mathematical Modelling, 2015, 39(15): 4257-4265.

[9] T. Söderström. Comparing some classes of bias-compensating least squares methods. Automatica, 2013, 49(3): 840-845.

[10] L. Ljung. System identification-theory for the user (2nd ed.). Upper Saddle River, NJ, USA: Prentice Hall (1999).

[11] S. Huffel. Recent Advances in Total Least Squares Techniques and Errors-in-Variables Modeling. Philadelphia, PA: SIAM (1997).

[12] S. Huffel, P. Lemmerling. Total Least Squares and Errors-in-Variables Modeling. Analysis, Algorithms and Applications. Dordrecht, The Netherlands: Kluwer (2002).

[13] B. Mu, H. Chen. Recursive identification of errors-in-variables Wiener systems. Automatica, 2013, 49(9): $2744-2753$.

[14] T. Söderström, Y. Irshad, M. Mossberg, W. Zheng. On the accuracy of a covariance matching method for continuous-time errors-in-variables identification. Automatica, 2013, 49(10): 2982-2993.

[15] Q. Song. Identification of errors-in-variables systems with nonlinear output observations. Automatica, 2013, 49(4): 987-992.

[16] T. Söderström. Errors-in-variables methods in system identification. Automatica, 2007, 43(6): 939-958.

[17] T. Söderström, M. Mossberg, M. Hong. A covariance matching approach for identifying errors-in-variables systems. Automatica, 2009, 45(9): 2018-2031.

[18] E. Bai. An optimal two-stage identification algorithm for Hammerstein-Wiener nonlinear systems. Automatica, 1998, 34(3): 333-338.

[19] X.G. Liu, Y.X. Zhou, L. Cong, F. Ding. High-purity control of internal thermally coupled distillation columns based on the nonlinear wave model. Journal of Process Control, 2011, 21(6): 920-926.

[20] J. Chen, L.X. Lv, R.F. Ding. Multi-innovation stochastic gradient algorithms for dual-rate sampled systems with preload nonlinearity. Applied Mathematics Letters, 2013, 26(1): 124-129.

[21] E. Malekshahi, S.M.A. Mohammadi. The model order reduction using LS, RLS and MV estimation methods. International Journal of Control, Automation, and Systems, 2014, 12(3): 572-581.

[22] D.Q. Wang, T. Shan, R. Ding. Data filtering based stochastic gradient algorithms for multivariable CARARlike systems. Mathematical Modelling and Analysis, 2013, 18(3): 374-385. 
[23] D.Q. Wang, F. Ding, X.M. Liu. Least squares algorithm for an input nonlinear system with a dynamic subspace state space model. Nonlinear Dynamics, 2014, 75(1-2): 49-61.

[24] H. Ase, T. Katayama. A subspace-based identification of Wiener-Hammerstein benchmark model. Control Engineering Practice, 2015, 44: 126-137.

[25] B.Q. Mu, H.F. Chen. Recursive identification of errors-in-variables Wiener-Hammerstein systems. European Journal of Control, 2014, 20(1): 14-23.

[26] M. Schoukens, A. Marconato, R. Pintelon, G. Vandersteen, Y. Rolain. Parametric identification of parallel Wiener-Hammerstein systems. Automatica, 2015, 51: 111-122. 